\title{
Protective effects and mechanisms of total alkaloids of Rubus alceaefolius Poir on non-alcoholic fatty liver disease in rats
}

\author{
HAIYIN ZHENG ${ }^{1}$, JINYAN ZHAO ${ }^{2}$, YUQING ZHENG ${ }^{1}$, JUAN WU ${ }^{3}$, \\ YAN LIU ${ }^{1}$, JUN PENG ${ }^{2}$ and ZHENFENG HONG ${ }^{1}$ \\ ${ }^{1}$ College of Integrative Medicine, Fujian University of Traditional Chinese Medicine, Fuzhou, Fujian 350122; \\ ${ }^{2}$ Academy of Integrative Medicine; ${ }^{3}$ Simulation Hospital, Fujian University of Traditional Chinese Medicine, \\ Fuzhou, Fujian 350122, P.R. China
}

Received August 3, 2013; Accepted May 9, 2014

DOI: $10.3892 / \mathrm{mmr} .2014 .2403$

\begin{abstract}
The plant Rubus alceaefolius Poir is used as a hepatic protectant in Traditional Chinese Medicine. The aim of the present study was to confirm the protective effect of the total alkaloids of Rubus alceaefolius Poir (TARAP) on the liver and to evaluate the potential molecular mechanisms associated with adipocytokines underlying non-alcoholic fatty liver disease (NAFLD) in rats. To generate the NAFLD model, Sprague-Dawley rats were administered a high-fat diet and following 12 weeks of model construction, rats were orally treated with a positive control drug and different doses of TARAP daily for 28 days. The rats were then sacrificed and the livers were collected to evaluate the liver index (LI) and observe histological changes by hematoxylin and eosin (H\&E) staining. The secretion levels of alanine aminotransferase (ALT) and aspartate aminotransferase (AST) in serum were examined by ELISA. Finally, the expression levels of leptin (LEP), resistin and adiponectin (APN) in liver tissues were determined by immunohistochemistry (IHC). The results demonstrated that, in the group treated with methionine and choline bitartrate tablets and in the groups treated with different doses of TARAP, there was a significant reduction in the $\mathrm{LI}(\mathrm{P}<0.05$ or $\mathrm{P}<0.01)$, a downregulation of the secretion levels of ALT and AST, reduced levels of LEP and resistin
\end{abstract}

Correspondence to: Professor Zhenfeng Hong, College of Integrative Medicine, Fujian University of Traditional Chinese Medicine, 1 Huatuo Road, Minhou Shangjie, Fuzhou, Fujian 350122, P.R. China

E-mail: hzflab@gmail.com

Abbreviations: TARAP, total alkaloids of Rubus alceaefolius Poir; NAFLD, non-alcoholic fatty liver disease; LI, liver index; SD, Sprague-Dawley; HE, hematoxylin-eosin; IHC, immunohistochemistry; LEP, leptin; APN, adiponectin; NASH, non-alcoholic steatohepatitis

Key words: Rubus alceaefolius Poir, total alkaloids, leptin, resistin, adiponectin and an increased expression of APN in the liver of NAFLD rats compared with the model group. Furthermore, the effect of TARAP treatment of NAFLD rats was dose dependent. In conclusion, TARAP is a potential agent for downregulating LEP and resistin and upregulating APN expression in rats with NAFLD. Furthermore, TARAP may be a potential candidate for improving treatment responses in patients with NAFLD.

\section{Introduction}

Non-alcoholic fatty liver disease (NAFLD) is a major cause of liver disease worldwide that encompasses a pathological spectrum ranging from simple steatosis and non-alcoholic steatohepatitis (NASH), to liver cirrhosis, hepatocellular carcinoma and liver failure. The prevalence of NAFLD is $~ 20-30 \%$ in western countries and $12-24 \%$ in the Asia-Pacific region and has thus become a major health problem worldwide (1).

Previously, several studies have demonstrated that NAFLD not only is a progressive liver disease, but is also a co-factor in other chronic liver diseases. It has been identified as part of the metabolic syndrome and is widely considered as a sign of systemic inflammatory status and cardiovascular disease $(2,3)$. Despite this, its underlying pathogenesis remains elusive and defining the mechanisms involved has become the focus of a number of studies $(4,5)$. Several hypotheses have been proposed as a result, including the well-accepted 'two-hit' theory by Day and James (6), which suggests that the liver is steatotic as a result of a 'first hit', namely lipid accumulation, and becomes vulnerable to secondary insults, including oxidative stress, adipocytokine and endotoxins, which promotes further hepatic injury. It is now hypothesized that the pathogenesis of NAFLD is also associated with insulin resistance, abnormal lipid metabolism, oxidative stress and lipid peroxidation (7).

Previous studies have revealed that adipose tissue-derived adipocytokines contribute to the pathogenesis of NAFLD. Adipocytokines, including leptin (LEP), adiponectin (APN) and resistin, as fat-derived proteins, have dual roles, including regulating the metabolism through endocrine, paracrine and autocrine mechanisms, as well as having inflammatory and immune regulatory functions (8). LEP is the main regulatory factor of adipose tissues. In the liver, LEP induces its anti-lipid effects by lowering the expression of hepatocyte steroid regu- 
latory element-binding protein-1 (SREBP-1) (9). Lack of or a high level of LEP may eventually result in the occurrence of fatty liver. LEP is also involved in the formation of fibrosis and has an important role in congenital and acquired immunity (10), regulating angiogenesis, wound healing and insulin sensitivity $(11,12)$. APN is an adipocyte-specific $28 \mathrm{kDa}$ secreted protein. It is expressed exclusively in adipose tissue and is an important regulator in the network of body lipid metabolism and glucose homeostasis. When APN forms a complex with its receptor, it has numerous important biological roles. APN has anti-diabetic, anti-obesity, anti-atherosclerotic and anti-inflammatory effects. Furthermore, it reduces body fat and improves liver and peripheral insulin sensitivity (13). While APN enhances insulin sensitivity, LEP, in contrast, has an inhibitory effect (14).

At present, no effective pharmacological therapy is available for patients with NAFLD, with Western treatment strategies focusing on the causes and associated factors. Traditional Chinese Medicine has a number of benefits and advantages in the treatment of NAFLD (15). Rubus alceaefolius Poir is a plant of the rosaceae rubus family and its root and leaf are employed as medicinal agents with effects including activating blood and eliminating stasis, dispersing heat and arresting pain (16). Rubus alceaefolius Poir is widely used in the clinic as an anti-inflammatory, antioxidant, antimutagenic, anticancer, antifungal, hypoglycemic, coagulant and antihaemorrhagic herb in China (17). The key components of Rubus alceaefolius Poir include alkaloids, flavonoids, terpenes, polysaccharides and tannins $(18,19)$. Preliminary studies identified that the total alkaloids of Rubus alceaefolius Poir (TARAP) had antioxidant and hepatic-injury protective effects in rats with NAFLD (20-22).

At present, TARAP is rarely utilized as a treatment strategy for NAFLD, and the protective effects of TARAP on NAFLD, possibly via the involvement of adipocytokines, to the best of our knowledge, have not yet been reported. Therefore, in the present study, the therapeutic effects of TARAP in a rat model of NAFLD were assessed, and the underlying molecular mechanisms were investigated.

\section{Materials and methods}

Materials and reagents. Rubus alceaefolius Poir was provided by the Pharmaceutical College of Fujian University of Traditional Chinese Medicine (TCM; Fuzhou, China). Rubus alceaefolius Poir was first collected and identified in the Anxi mountainous regions of Fujian (China) by Professor Lu Wei of Fujian University of TCM. The voucher specimen was deposited to the herbarium of pharmaceutical college of Fujian University of TCM with the registration no. 20070126. The plants were dried and stored for 1 year.

Lard oil and pig bile salts were purchased from Hui Xing Biochemical Reagent Co., Ltd. (Shanghai, China). Methionine and choline bitartrate (CMCB) capsules were purchased from Sanofi-Aventis Pharmaceutical Co., Ltd. (Beijing, China). APN (rabbit polyclonal antibody) and APN receptor-2 (ADI-R-2, rabbit polyclonal antibody), LEP (rabbit polyclonal antibody) and LEP receptor (rabbit polyclonal antibody), visfatin and resistin (rabbit polyclonal antibody) were obtained from Bohai Biotechnology Development Co., Ltd. (Hebei, China). For the analysis of serum alanine aminotransferase (ALT) and asparagine aminotransferase (AST) secretion, an ALT and AST test kit was purchased from Nanjing Jiancheng Bioengineering Institute (Nanjing, Jiangsu, China). All other chemicals, unless otherwise stated, were obtained from Sigma Chemicals (St. Louis, MO, USA).

Animals. Fifty specific pathogen-free grade adult Sprague-Dawley (SD) rats with a body weight of 180-220 g, purchased from Shanghai Si-Lai-Ke Experimental Animal Co. Ltd. (Shanghai, China), were housed under controlled temperature $\left(21-23^{\circ} \mathrm{C}\right)$, humidity, a 12-h light/day cycle and with free access to a standard rat diet and tap water.

All animal experiments were conducted in compliance with international ethical guidelines and the National Institutes of Health Guide concerning the Care and Use of Laboratory Animals and approved by the Animal Care and Use Committee of Fujian University of TCM.

Preparation of TARAP. TARAP was extracted as described previously (23). The root samples of Rubus alceaefolius Poir (10 g) were cold-macerated with a $500 \mathrm{ml}$ chloroform/methanol/ammonia solution (15:4:3) for $2 \mathrm{~h}$, then extracted utilizing an ultrasonic method for $30 \mathrm{~min}$. Then, the mixture was cooled and was filtered, concentrated and dessicated. The resultant residue was dissolved in $100 \mathrm{ml} 2 \%$ sulfuric acid solution and filtered, washed with $20 \mathrm{ml} 2 \%$ sulfuric acid and acetate buffer (pH 3.6). Finally, the solution was dried to powder and kept for further use. Total alkaloid content was measured by acid dye colorimetry (22). The tests were performed in triplicate and the average total alkaloid content obtained was $80.03 \mathrm{mg}$.

Groups and treatment. A total of $50 \mathrm{SD}$ rats were equally divided into five groups at random $(n=10)$; namely the control group (Control), model group (Model), positive control group, CMCB and low (TARAP-L) and high (TARAP-H) dose groups of TARAP. The rat model of NAFLD was induced by feeding a high-fat diet which containing $10 \%$ lard oil, $2 \%$ cholesterol and $0.7 \%$ pig bile salts. Besides the control group, which was fed the basic diet and corn flour, the other groups received the high-fat diet. Following eight weeks, histopathological examination proved that the NAFLD model had been successfully established. From the ninth week, the control and model groups were administered distilled water at dose of $10 \mathrm{ml} / \mathrm{kg}$ body weight each day, the positive control groups were given CMCB at dose of $0.35 \mathrm{~g} \mathrm{Kg}^{-1}$ and TARAP groups were given a dose of $0.36 \mathrm{~g} / \mathrm{kg}$ and $1.44 \mathrm{~g} / \mathrm{kg}$ (low- and high-dose alkaloid groups, respectively). The medicines were orally administered daily for 28 consecutive days. The rats were weighed weekly during the experiments.

Sample collection. A total of $24 \mathrm{~h}$ following the last administration, rats were anesthetized with sodium pentobarbital (50 $\mathrm{mg} / \mathrm{kg}$, i.p.), the abdominal aorta was exposed through a midline abdominal incision and blood was collected into a sodium citrate tube. Serum was obtained by centrifugation at $3,500 \mathrm{x} \mathrm{g}$ for $15 \mathrm{~min}$ at $4^{\circ} \mathrm{C}$ and stored at $-80^{\circ} \mathrm{C}$. Following this, the liver was extracted and weighed. The liver index (LI) was calculated as: Liver weight (LW)/body weight (BW) $\mathrm{x} 100 \%$. A part of the right liver lobe of each rat was fixed 
Table I. Effect of TARAP treatment on BW, LI and fat content.

\begin{tabular}{lcccccc}
\hline Groups & Dose $\left(\mathrm{g} \mathrm{Kg}^{-1}\right)$ & Initial BW $(\mathrm{g})$ & Final BW $(\mathrm{g})$ & LW & LI & Fat content \\
\hline Control & - & $234.410 \pm 16.981$ & $403.300 \pm 106.902$ & $10.749 \pm 3.114$ & $0.025 \pm 0.002$ & $3.848 \pm 0.204$ \\
Model & - & $239.502 \pm 19.693$ & $592.267 \pm 104.114$ & $20.259 \pm 6.504^{\mathrm{a}}$ & $0.039 \pm 0.005^{\mathrm{a}}$ & $30.156 \pm 2.611^{\mathrm{a}}$ \\
CMCB & 0.5 & $230.611 \pm 14.433$ & $437.378 \pm 100.037$ & $14.073 \pm 4.065^{\mathrm{b}}$ & $0.035 \pm 0.004^{\mathrm{b}}$ & $20.656 \pm 3.910^{\mathrm{c}}$ \\
TARAP-L & 0.36 & $232.583 \pm 21.146$ & $426.612 \pm 116.831$ & $19.559 \pm 6.747$ & $0.039 \pm 0.005$ & $20.105 \pm 2.141^{\mathrm{c}}$ \\
TARAP-H & 1.44 & $236.83 \pm 15.22$ & $433.283 \pm 75.474$ & $13.857 \pm 3.470^{\mathrm{b}}$ & $0.034 \pm 0.006^{\mathrm{b}}$ & $20.002 \pm 2.602^{\mathrm{c}}$ \\
\hline
\end{tabular}

${ }^{\mathrm{a}} \mathrm{P}<0.01$ for the difference between model and control groups. ${ }^{\mathrm{b}} \mathrm{P}<0.05,{ }^{\mathrm{C}} \mathrm{P}<0.01 \mathrm{vs}$. model group. TARAP, total alkaloids of Rubus alceaefolius Poir; BW, body weight; LI, liver index; LW, liver weight; CMCB, compound methionine and choline bitartrate; TARAP-L; low dose TARAP; TARAP-H, high-dose TARAP.

in $10 \%$ formalin solution for histopathological examination and immunohistochemistry. The other part of the liver was snap-frozen in liquid nitrogen and stored at $-8^{\circ} \mathrm{C}$.

Fat content determination. The fresh hepatic tissue was dried to a constant weight. The sample was mashed and ground until it formed a homogenate. A total of $2 \mathrm{~g}$ of the sample was placed in a soxhlet extractor and absolute ether was poured into the flask. The flask was placed in a heated water bath of the concentrator apparatus. The solvent was removed by applying a steady stream of nitrogen over the sample. Once all of the solvent had been removed, the flask was dried on the outside surface to remove water and weighed to determine the mass of extracted fat. From this value, the fat content of the original liver sample was calculated.

Biochemical assay. Serum levels of ALT and AST were measured using an ELISA kit (Nanjing Jiancheng Bioengineering Institute, Nanjing, Jiangsu, China) according to the manufacturer's instructions and the absorbance was measured by an ELISA reader (Model ELX800; BioTek Instruments, Inc., Winooski, VT, USA).

Histological examination. The fixed hepatic tissue was dehydrated in graded ethanols, embedded in paraffin, sliced into serial $5 \mu \mathrm{m}$ sections, deparaffinized in xylene, rehydrated in graded ethanol and then stained with hematoxylin and eosin (H\&E) for histological observation under a light microscope (11090137001; Leica Microsystems CMS Gmbh, Mannheim, Germany).

Immunohistochemical (IHC) analysis. Sections of $5 \mu \mathrm{m}$ thickness were sliced, deparaffinized, rehydrated, submerged in $1 \%$ hydrogen peroxide, epitope retrieved, soaked in goat serum followed by incubation with primary antibodies overnight at $4^{\circ} \mathrm{C}$. The control slides were incubated with phosphate-buffered saline (PBS) without primary antibodies against APN, ADI-R 2, LEP, LEP receptor and resistin. The slides were then washed in PBS and incubated for $20 \mathrm{~min}$ at $37^{\circ} \mathrm{C}$ with biotinylated secondary antibody followed by conjugated horseradish peroxidase-labeled streptavidin. Following rinsing in PBS, the slides were exposed to streptavidin biotin-peroxidase complex for $20 \mathrm{~min}$ at $37^{\circ} \mathrm{C}$ and again rinsed in PBS. The slides were stained with DAB (3,3'-diaminobenzidine) color develop- ment solution for $6 \mathrm{~min}$, then rinsed and counterstained with hematoxylin. The IHC slides were examined with a method of immunohistochemical scoring (IHS), which was calculated by combining an estimate of the percentage of immunoreactive cells (quantity score) with an estimate of the staining intensity (staining intensity score), as follows: No staining was scored as $0,1-10 \%$ of cells stained scored as $1,11-50 \%$ as $2,51-80 \%$ as 3 and $81-100 \%$ as 4 . Staining intensity was rated on a scale of $0-3$, with 0 , negative; 1 , weak; 2 , moderate and 3 , strong. The raw data were converted to the IHS by multiplying the quantity and staining intensity scores (24).

Statistical analysis. All quantitative experiments were performed more than three times. Data are expressed as the mean \pm standard deviation. Statistical analyses were by one-way analysis of variance performed using SPSS 16.0 (SPSS, Inc., Chicago, IL, USA). A student's t-test was used to determine the significant differences between the groups. $\mathrm{P}<0.05$ was considered to indicate a statistically significant difference.

\section{Results}

Effect of TARAP on the BW and LI. The high-fat diet caused a progressive increase in the body weight of the NAFLD rats. The mean body weight following nine weeks of high-fat diet feeding was $>2 x$ the weight at the beginning of the feeding. The high-fat diet significantly increased the liver weight $(\mathrm{P}<0.01)$ and the $\mathrm{LW} / \mathrm{BW}$ ratio $(\mathrm{P}<0.01)$ when compared with the rats receiving the normal diet. TARAP-L/TARAP-H treatment significantly reduced the BW gain, LW and LI as compared with those of the model group rats (Table I).

TARAP reduces the fat content of the liver. Compared with the normal diet rats, the high-fat diet significantly increased the serum fat content of the liver $(\mathrm{P}<0.01)$. TARAP-L and TARAP-H treatment markedly reduced the fat content of the liver $(\mathrm{P}<0.01)$ as compared with the high-fat diet rats (Table I).

TARAP ameliorates the fatty degeneration of liver tissue. Photomicrographs of the liver samples stained with H\&E are shown in Fig. 1. In the normal group, the liver was normal without any pathological symptoms (Fig. 1A). In these specimens, there was a high preservation of hepatocytes and the lining cells of both sinusoids and postinusoidal venules, as well 


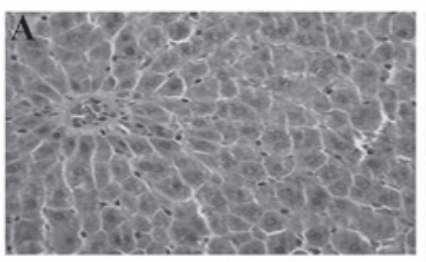

Control

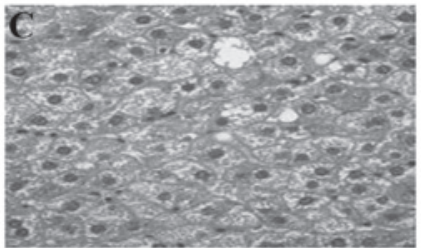

CMCB

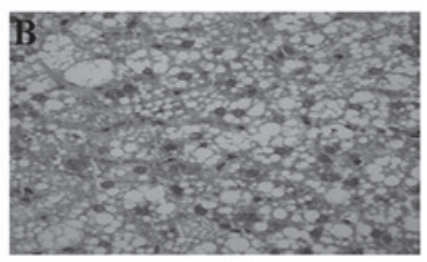

Model

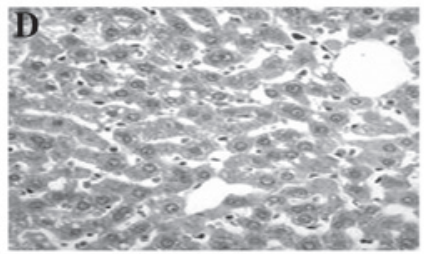

TARAP-L

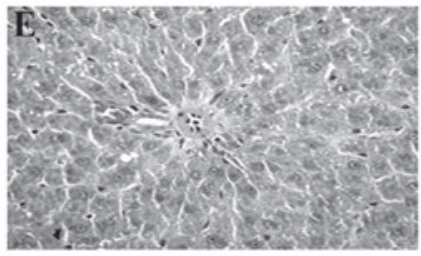

TARAP-H

Figure 1. Effect of TARAP treatment on the histopathological features of the liver tissue. Samples were stained with H\&E and observed under the microscope. Representative images were captured at a magnification of x100. (A) Rats fed an ordinary diet for 12 weeks were normal and did not exhibit any pathological symptoms. (B) Significant steatosis and inflammatory cells appeared in the livers of the rats fed a high-fat diet. (C, D, E and F) Degenerative changes of hepatic lesions of NAFLD were observed in the CMCB and TARAP-treated rats. TARAP, total alkaloids of Rubus alceaefolius Poir; CMCB, compound methionine and choline bitartrate; TARAP-L; low dose TARAP; TARAP-H, high-dose TARAP; H\&E, hematoxylin and eosin.

Table II. Effect of TARAP on the levels of AST and ALT.

\begin{tabular}{lcccc}
\hline Groups & $\mathrm{n}$ & Dose $\left(\mathrm{g} \mathrm{Kg}^{-1}\right)$ & ALT $\left(\mathrm{U} \mathrm{L}^{-1}\right)$ & AST $\left(\mathrm{U} \mathrm{L}^{-1}\right)$ \\
\hline Control & 10 & - & $32.30 \pm 4.57$ & $165.21 \pm 37.84$ \\
Model & 9 & - & $48.33 \pm 7.01^{\mathrm{a}}$ & $251.59 \pm 65.98^{\mathrm{a}}$ \\
CMCB & 10 & 0.5 & $40.69 \pm 7.42^{\mathrm{b}}$ & $222.42 \pm 54.18$ \\
TARAP-L & 10 & 0.36 & $37.95 \pm 5.43^{\mathrm{c}}$ & $240.32 \pm 43.27$ \\
TARAP-H & 9 & 1.44 & $36.23 \pm 8.51^{\mathrm{c}}$ & $187.99 \pm 48.51^{\mathrm{b}}$
\end{tabular}

${ }^{\mathrm{a}} \mathrm{P}<0.01$ for the difference between model and control groups. ${ }^{\mathrm{b}} \mathrm{P}<0.05$, ${ }^{\mathrm{C}} \mathrm{P}<0.01$ vs. the model group. TARAP, total alkaloids of Rubus alceaefolius Poir; $\mathrm{CMCB}$, compound methionine and choline bitartrate; TARAP-L; low dose TARAP; TARAP-H, high-dose TARAP.

as structural integrity of the hepatic lobule. In the hepatocytes of the high-fat diet model rats, numerous large vacuoles within a number of lipid droplets were observed, as well as mononuclear cell infiltration, picnotic nuclei and the rupturing in the endothelium of certain central veins (Fig. 1B). In the CMCB group, the lobulation of the liver was distinct, there were a number of empty lipocytes that were mainly small vacuoles, which accounted for $\sim 1 / 3$ of hepatic lobules (Fig. 1C). The degenerative changes of such hepatic lesions of NAFLD were observed in the TARAP-treated rats (Fig. 1D and E). In the TARAP-H group, the morphology of the liver was similar to that in the

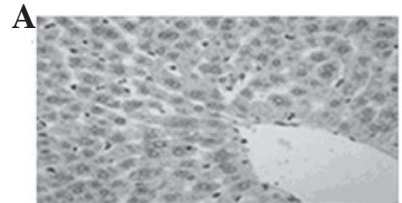

Control

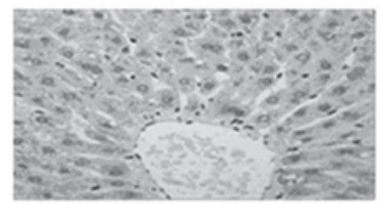

TARAP-L

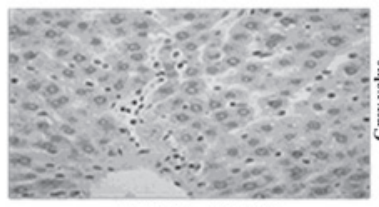

TARAP-H

\section{B}

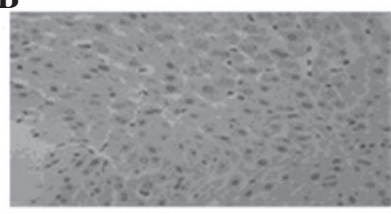

Control

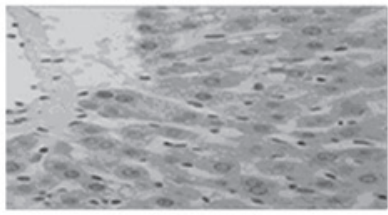

TARAP-L

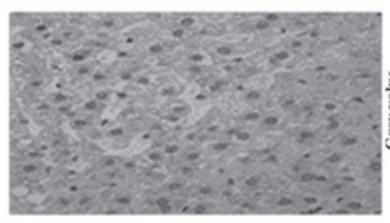

TARAP-H

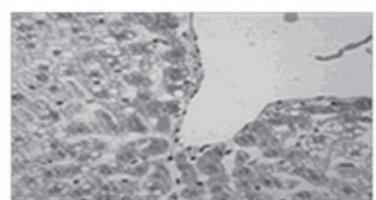

Model

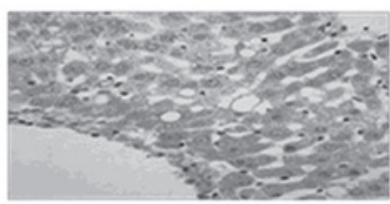

CMCB
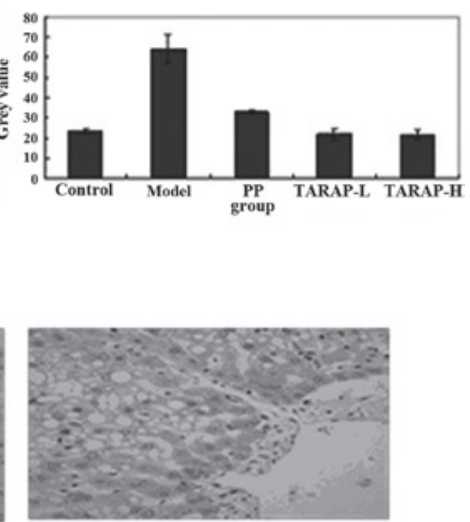

Model

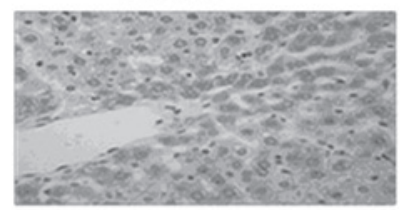

Leptin-R

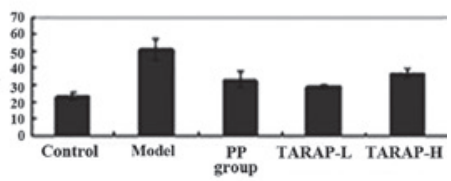

Figure 2. Effect of TARAP treatment on the protein expression of LEP and its receptor in liver tissue. (A) Protein expression of LEP in different groups was observed using IHC staining (magnification, x400). (B) Protein expression of the LEP receptor in different groups was observed using IHC staining (magnification, $\mathrm{x} 400$ ). Data are presented as the mean \pm standard deviation (error bars). TARAP, total alkaloids of Rubus alceaefolius Poir; CMCB, compound methionine and choline bitartrate; TARAP-L; low dose TARAP; TARAP-H, high-dose TARAP; LEP, leptin; IHC, immunohistochemistry.

CMCB group, but it accounted for $<1 / 3$ of the hepatic lobule. In TARAP-L group, the lobulation was distinct in the liver, there were a number of middle or small vacuoles, accounting for $\sim 1 / 3-1 / 2$ of the hepatic lobule. TARAP treatment ameliorated the histopathological changes observed in the NAFLD liver in a dose-dependent manner.

Effect of TARAP on the levels of AST and ALT. The ELISA data summarized in Table II revealed that the levels of ALT in TARAP-L and TARAP-H were lower compared with those in the model group $(\mathrm{P}<0.05)$. The levels of AST in TARAP-L 
A

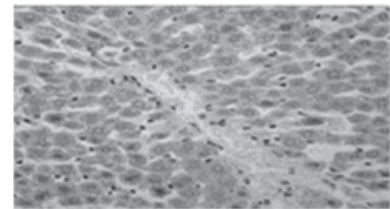

Control

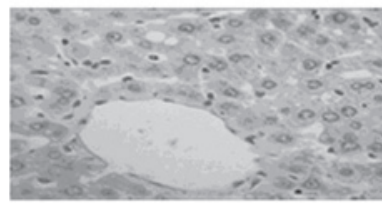

TARAP-L

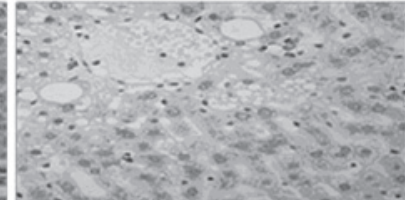

Model

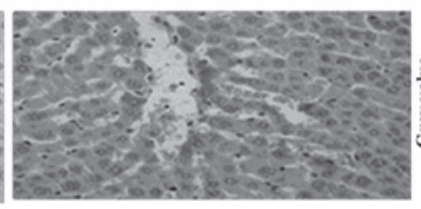

TARAP-H

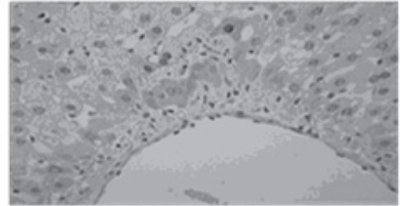

CMCB

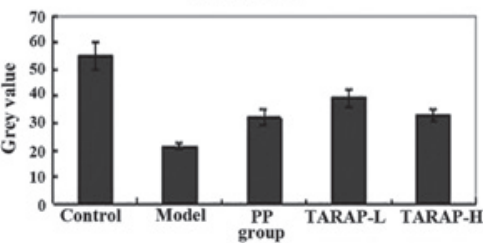

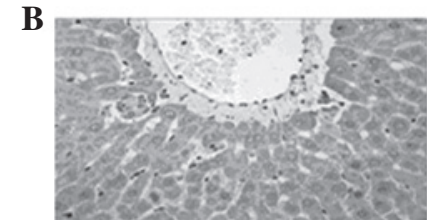

Control

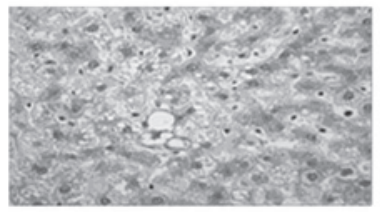

TARAP-L

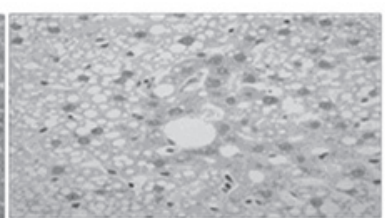

Model

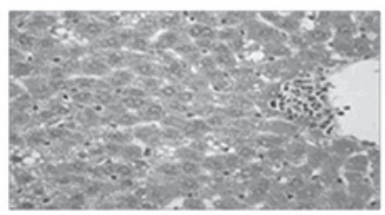

TARAP-H

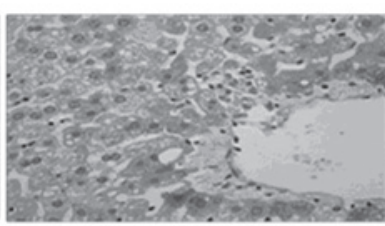

CMCB

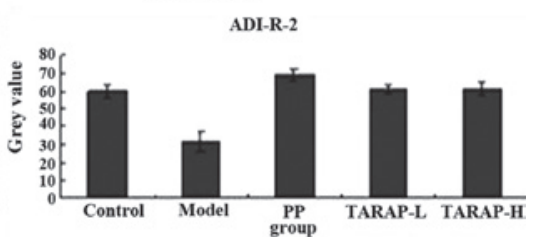

Figure 3. Effect of TARAP treatment on the protein expression of APN and its receptor ADI-R-2 in liver tissue. (A) Protein expression of APN in different groups was observed using IHC staining (magnification, x400). (B) Protein expression of the APN receptor in different groups was observed using IHC staining (magnification, x400). Data are presented as the mean \pm standard deviation (error bars). TARAP, total alkaloids of Rubus alceaefolius Poir; APN, adiponectin; CMCB, compound methionine and choline bitartrate; TARAP-L; low dose TARAP; TARAP-H; high-dose TARAP; IHC, immunohistochemistry.

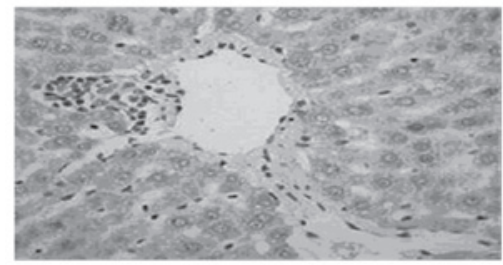

Control

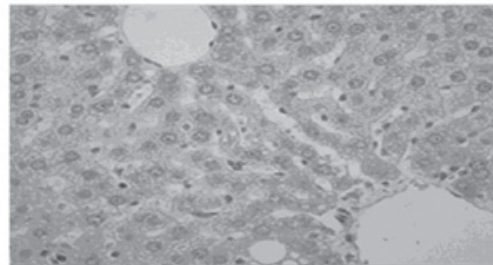

TARAP-L

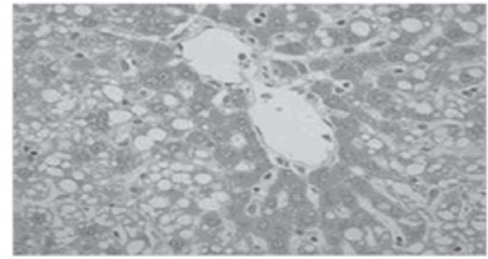

Model

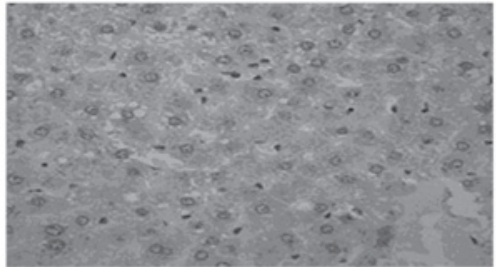

TARAP-H

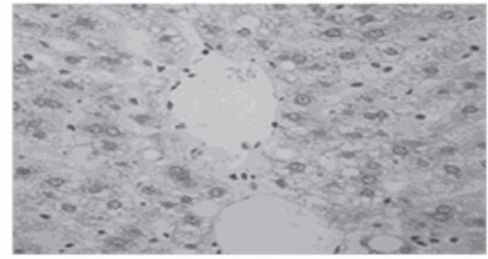

CMCB

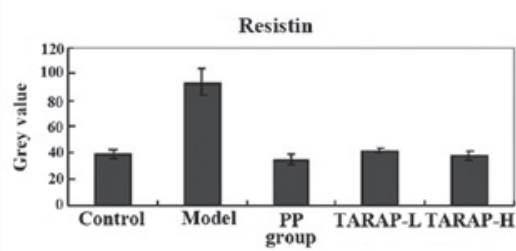

Figure 4. Effect of TARAP treatment on the protein expression of resistin in liver tissue. Protein expression of resistin in different groups was observed using immunohistochemical staining (magnification, x400). Data are expressed as the mean \pm standard deviation (error bars). TARAP, total alkaloids of Rubus alceaefolius Poir; CMCB, compound methionine and choline bitartrate; TARAP-L; low dose TARAP; TARAP-H; high-dose TARAP.

and TARAP-H were decreased, but only the TARAP-H group exhibited a significant difference compared with the model group $(\mathrm{P}<0.05)$.

TARAP regulates the expression of adipocytokines. The expression of LEP and LEP receptor, APN and APN receptor, and resistin in the liver, and the respective grey values, which were obtained by the scoring system mentioned previously, are shown in Figs. 2-4. Compared with the normal group, LEP levels increased in the model rats, while APN and resistin decreased, with a marked difference $(\mathrm{P}<0.01)$. In the administration group, LEP evidently decreased $(\mathrm{P}<0.01)$ and APN and resistin increased, which was a statistically significant difference compared with the model group $(\mathrm{P}<0.05)$. 


\section{Discussion}

Feeding rats with a high-fat diet most commonly results in the development of NAFLD, which represents the typical pathological changes observed in human NAFLD patients. This model was utilized in the present study, where the rats exhibited typical hepatic lesions of NAFLD, including hepatomegaly, liver steatosis and inflammation $(25,26)$. Due to these characteristics, this method has been considered a useful and reliable tool for modelling NAFLD.

NAFLD may in part involve adipocytes, for it is a component of the metabolic syndrome and its metabolism is mediated by a complex network of mediators derived mainly from adipocytes, i.e. adipocytokines. There is increasing evidence that mediators released from the adipose tissue in obese subjects, including adipocytokines and classical cytokines, are key contributors to NAFLD (27). Adipocytokines, including adiponectin, LEP and resistin, are considered the most abundant adipocytokines produced in adipose tissue. LEP and its functional receptor are important in hepatic responses. LEP exerts crucial effects on glucose homeostasis and is capable of reversing hyperglycemia (28). In accordance with previous results, in the present study, LEP levels were increased in model rats as compared with those in the normal group $(\mathrm{P}<0.01)$, and following TARAP treatment, LEP levels decreased markedly $(\mathrm{P}<0.01)$, liver pathology improved and LI decreased. Therefore, improving LEP sensitivity therapeutically may enhance LEP and insulin resistance. In a previous study, it was reported that the expression of APN was lower, and its serum levels in NAFLD patients were reduced and exhibited distinct insulin resistance (29). In the present study, the model group exhibited significantly lower than normal levels of serum APN $(\mathrm{P}<0.01)$ by gastric perfusion of the high fat diet, as was consistent with previous studies. Following administration of TARAP, APN was upregulated, the liver pathology improved and the LI decreased. These results indicated that high APN levels contributed to NAFLD LI and liver pathology. This effect may be associated with APN's ability to promote liver and peripheral insulin sensitivity, reduce serum fatty acid levels and increase muscle fatty acid oxidation. Resistin has opposing effects on vascular endothelial cells as compared with adiponectin. Furthermore, it has been reported that resistin leads to insulin resistance, and hyperresistinemia increases blood glucose and insulin levels in mice (30).

In consideration of all the experimental results, it was suggested that the mechanism of action of TARAP in NAFLD may occur via the regulation of adipocytokines and enhancing insulin sensitivity. Furthermore, these data confirmed that TARAP has a number of beneficial effects on NAFLD. Further studies are required to further elucidate the molecular mechanisms of these beneficial effects.

The present study demonstrated that oral administration of TARAP had significant protective effects on NAFLD, which was evident from decreased LI, suppressed LEP and resistin levels and upregulated APN in NAFLD rats following TARAP treatment. The underlying mechanisms may be partially explained by the regulation of adipocytokines and it is suggested that TARAP may have potential clinical applications in the treatment of NAFLD.

\section{Acknowledgements}

This study was supported by the Natural Science Foundation of Fujian Province of China (no. 2010J01191), the major projects of Science and Technology Bureau of Fujian province (no. 2010Y2004; Fuzhou, Fujian, China) and The B class project of Fujian University of Traditional Chinese Medicine (XB2011006).

\section{References}

1. Fan JG and Farrell GC: Epidemiology of non-alcoholic fatty liver disease in China. J Hepatol 50: 204-210, 2009.

2. Everhart JE and Bambha KM: Fatty liver: think globally. Hepatology 51: 1491-1493, 2010.

3. Bruce KD and Byrne CD: The metabolic syndrome: common origins of a multifactorial disorder. Postgraduate Med J 85: 614-621, 2009.

4. Malaguarnera M, Rosa M Di, Nicoletti F and Malaguarnera L: Molecular mechanisms involved in NAFLD progression. J Mol Med (Berl) 87: 679-695, 2009.

5. Adams LA, Angulo P and Lindor KD: Nonalcoholic fatty liver disease. CMAJ 172: 899-905, 2005.

6. Day CP and James OF: Steatohepatitis: a tale of two 'hits'? Gastroenterology 114: 842-845, 1998.

7. Guzman G, Brunt EM, Petrovic LM, Chejfec G, Layden TJ and Cotler SJ: Does nonalcoholic fatty liver disease predispose patients to hepatocellular carcinoma in the absence of cirrhosis? Arch Pathol Lab Med 132: 1761-1766, 2008.

8. Yan E, Durazo F, Tong M and Hong K: Nonalcoholic fatty liver disease: pathogenesis, identification, progression and management. Nutr Rev 65: 376-384, 2007.

9. Kakuma T, Lee Y, Higa M, Wang ZW, Pan W, Shimomura I and Unger RH: Leptin, troglitazone and the expression of sterol regulatory element binding proteins in liver and pancreatic islets. Proc Natl Acad Sci USA 97: 8536-8541, 2000.

10. Matarese G, Moschos S and Mantzoros CS: Leptin in immunology. J Immunol 174: 3137-3142, 2005.

11. Murad A, Nath AK, Cha ST, Demir E, Flores-Riveros J and Sierra-Honigmann MR: Leptin is an autocrine/paracrine regulator of wound healing. FASEB J 17: 1895-1897, 2003.

12. Mantzoros CS: The role of leptin in human obesity and disease: a review of current evidence. Ann Internal Med 130: 671-680, 1999.

13. Shklyaev S, Aslanidi G, Tennant M, et al: Sustained peripheral expression of transgeneadiponectin offsets the development of diet-induced obesity in rats. Proc Natl Acad Sci USA 100: 14217-14222, 2003.

14. Yalniz M, Bahcecioglu IH, Ataseven H, Ustundag B, Ilhan F, Poyrazoglu OK and Erensoy A: Serum adipokine and ghrelin levels in nonalcoholic steatohepatitis. Mediators Inflamm 2006: 34295, 2006.

15. Li JX, Chen RH, Su DM and L Li : Advances in management of nonalcoholic fatty liver disease by Chinese medicine. World Chin J Digestol 18: 1443-1451, 2010.

16. Yao ZS and Yang WL: The Rubus medicinal plants in Jiangxi and suggestion of utilization. J. Chin Med Mater 18: 551-554, 1995.

17. Xu PJ, Tan MX and XM Chen: New progress of the Rubupharmacological effects. Health Vocational Educ 21: 145-146, 2003.

18. Gan L, Wang B, Liang H, Zhao YY and Jiang FC: Chemical constituents from Rubus alceaefolius Poir. J Beijing Med Univ 32: 226-228, 2000.

19. Meng XJ, Liu B, Re ZCD, She GM and Jiang YY: Progress of chemical constituents and pharmacology of genus Rubus. Nat Prod Res Dev 23: 767-775, 2011.

20. Zheng HY, Zhao JY, Liu Y, Zheng YQ, Wu J and Hong ZF: Effect of total alkaloids of Rubus alceaefolius on oxidative stress in rats with non-alcoholic fatty liver disease. China J Chin Materia Medica 36: 2383-2387, 2011.

21. Zhao JY, Zheng YQ, Zheng HY, Zhong XY, Wu J and Hong ZF: Anti-oxidation of total alkaloids from Rubus alceaefolius Poir on nonalcoholic fatty liver. J Fujian Univ Traditional Chin Med 21: 15-17, 2011.

22. Zhao JY, Wu ZS, Chen W and Hong ZF: Study of protective effect of liver injury of total alkaloids from Rubus alceaefolius Poir from different part. Lishizhen Med Materia Medica Res 21: 2186-2188, 2010 
23. Hong ZF, Xu W, Li TJ, Zhou JH and Hu J: Determination of total alkaloids from the root of Rubus alceaefolius Poir. J Fujian Univ Traditional Chin Med 18: 28-30, 2008.

24. Soslow RA, Dannenberg AJ, Rush D, Woerner BM, Khan KN, Masferrer J and Koki AT : Cox-2 is expressed in human pulmonary, colonic and mammary tumors. Cancer 89: 2637-2645, 2000

25. Matteoni C, Younossi ZM, Gramlich T, Boparai N, Liu YC and McCullough AJ: Nonalcoholic fatty liver disease: a spectrum of clinical and pathological severity. Gastroenterology 116: 1413-1419, 1999.

26. Lieber CS, Leo MA, Mak KM, Xu YQ, Cao Q, et al: Model of nonalcoholic steatohepatitis. Am J Clin Nutr 79: 502-509, 2004.
27. Tilg H: Adipocytokines in nonalcoholic fatty liver disease: Key players regulating steatosis, inflammation and fibrosis. Curr Pharm Des 16: 1893-1895, 2010.

28. Heymsfield SB, Greenberg AS, Fujioka K, et al: Recombinant leptin for weight loss in obese and lean adults: a randomized, controlled, dose-escalation trial. JAMA 282: 1568-1575, 1999.

29. Jarrar MH, Baranova A, Collantes R, Ranard B, Stepanova M, et al: Adipokines and cytokines in non-alcoholic fatty liver disease. Aliment Pharmacol Ther 27: 412-421, 2008.

30. Banerjee RR, Rangwala SM, Shapiro JS, et al: Regulation of fasted blood glucose by resistin. Science 303: 1195-1198, 2004. 